American Journal of Applied Sciences 5 (8): 1005-1012, 2008

ISSN 1546-9239

(C) 2008 Science Publications

\title{
Determination of Ibuprofen and Paracetamol in Binary Mixture Using Chemometric-Assisted Spectrophotometric Methods
}

\author{
Wafaa S. Hassan \\ Department of Analytical Chemistry, Faculty of Pharmacy, \\ Zagazig University, Zagazig, Egypt
}

\begin{abstract}
Three methods are presented for simultaneous determination of ibuprofen and paracetamol without previous separation. The first method depends on first derivative UV spectrophotometry, with zero-crossing measurement. The first derivative amplitudes at 230 and $290 \mathrm{~nm}$ were selected for the assay of ibuprofen and paracetamol, respectively. The second method depends on first derivative of the ratio-spectra by measurements of the amplitudes at 280 and $290 \mathrm{~nm}$ for ibuprofen and paracetamol, respectively. Calibration graphs were established in the range of 5-100 and 10-100 $\mu \mathrm{g} \mathrm{mL} \mathrm{m}^{-1}$ for ibuprofen and paracetamol, respectively. The third method is the use of multivariate spectrophotometric calibration for the simultaneous determination of the analyzed mixture. The resolution of the studied binary mixture has been accomplished by using partial least squares (PLS) regression analysis. Although the components show an important degree of spectral overlap, they have been simultaneously determined with high accuracy, with no interference from pharmaceutical dosage form excipients. A comparison is presented with the related multivariate method of classical least squares (CLS) analysis, which is shown to yield less reliable results due to severe spectra overlap presented by the studied compounds. All of the proposed methods have been extensively validated. These methods allow a number of cost and time saving benefits. The described methods can be readily utilized for analysis of pharmaceutical formulations. There was no significant difference between the performance of all of the proposed methods regarding the mean values and standard deviations.
\end{abstract}

Key words: Ibuprofen, paracetamol, first derivative spectrophotometry, ratio derivative spectrophotometry, multvariate, pharmaceuticals

\section{INTRODUCTION}

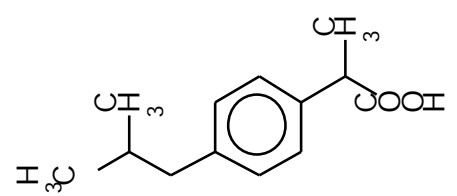

Ibuprofen is $\mathrm{a}^{[1]}$. The most recent methods chromatographic $^{[2-5]}, \quad$ electrochemical $^{[6,7]}$ and spectrophotometric ${ }^{[8-13]}$ techniques.

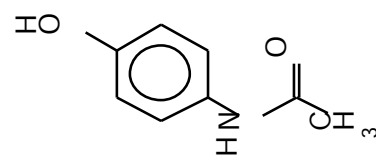

Paracetamol is an analgesic-antipyretic agent. It is effective in treating mild to moderate pain such as headache, neuralgia and pain of musculo-skeletal origin $^{[1]}$. The most recent methods for determination of paracetamol included chromatographic ${ }^{[14-16]}$, electrochemical $^{[17-19]}$ and spectrophotometric ${ }^{[20-22]}$ techniques.

Recently, derivative spectrophotometry has been found to be a useful method in the determination of mixtures with two or more components having overlapping spectra and in eliminating interference from formulation matrix by using the zero-crossing techniques ${ }^{[23-26]}$.

Furthermore, ratio-spectra derivative spectrophotometric method has also been found to be useful in the estimation of drugs in their mixtures ${ }^{[27-29]}$. This method permits the determination of a component in their mixture at the wavelengths corresponding to a maximum or minimum and also the use of the peak-topeak between consecutive maximum and minimum. The main advantage of derivative of the ratio-spectra method may be the chance of easy measurements in correspondence of peaks so it permits the use of the wavelength of highest value of analytical signals 
(maximum or minimum). Moreover, the presence of a lot of maxima and minima is another advantage by the fact that these wavelengths give an opportunity for the determination of active compounds in the presence of other active compounds and excipients which possibly interfere the analysis.

Multivariate calibration methods applied to spectral data are being increasingly used for pharmaceutical analysis. Classical least squares ( CLS ) and Principal components regression (PCR) analysis are the most simplest multivariate methods that can be performed with easily accessible statistical software ${ }^{[30-33]}$.

CLS technique assumes that responses (absorbance) at each frequency (wavelengths) are proportional to component concentration units.

Model errors are assumed to derive from the measurement of spectral absorbance. So CLS requires that all interfering chemical components be known and included in the calibration data set. CLS has the advantage of improved precision when using many frequencies, due to signal averaging.

Calibration is realized by recording the spectra at $\mathrm{n}$-wavelengths of $\mathrm{m}$ standard mixtures, of known composition of c components. The spectra (absorbance or emission) are arranged into the columns of matrix $\mathrm{Y}$ (dimensions $\mathrm{n} \times \mathrm{m}$ ), with the composition of each mixture forming the columns of concentration matrix $\mathrm{X}$ (c x m)

$$
\mathrm{Y}=\mathrm{K} \cdot \mathrm{X}
$$

With a prior knowledge of $X$ and by recording data for $Y$, then the matrix of sensitivities, $K$, can be calculated, but after the rearrengmentof equation 1 to the following equation by multiplying the equation components by $\mathrm{X}^{\mathrm{t}}$ value as:

$$
\begin{aligned}
& Y \cdot X^{t}=K \cdot X \cdot X^{t} \\
& K=\left(X \cdot X^{t}\right)^{-1} \cdot Y \cdot X^{t}
\end{aligned}
$$

To avoid being under-determined, there must be measurements at more wavelengths than there are components (i.e. $\mathrm{n}=\mathrm{c}$ ). If $\mathrm{n}>\mathrm{c}$ then the component concentrations in an unknown mixture are obtained from its spectrum by,

$$
\mathrm{X}_{\text {unknown }}=\left(\mathrm{K}^{\mathrm{t}} \cdot \mathrm{K}\right)^{-1} \cdot \mathrm{K}^{\mathrm{t}} \mathrm{y}_{\text {unknown }}
$$

This CLS method is intuitively appealing since it is based on some generally assumed relationship, e.g., Beer's law and it can be used for moderately complex composition of the calibration mixtures, i.e. the concentration of each absorbing species. PCR is a twostep procedure, in the first step, one estimating the number of principal components by one or more of the following criteria, the percentage of explained variance, eigen value-one criterion, the Scree-test and Cross validation. They can be considered as new variables that summarize in an optimal way the variation present in the spectra, in the second step, CLS is applied to the newly obtained latent variables. When co-linearity between original variables occurs, principal component plots often allow better interpretation of the variations observed in the data set than plots of original variables selected by CLS. As modeling method, it is less performant than CLS when performing prediction within the calibration domain and when the model is indeed linear. It is more reliable if extrapolation may be required. It is a linear method, but it is able to perform quite well for moderately nonlinear data. As CLS, it is a global method ${ }^{[34-36]}$.

The purpose of the present study was to investigate the utility of derivative, derivative ratio spectrophotometry and multivariate techniques in the assay of formulations of ibuprofen with paracetamol in certain analgesic binary mixture. The results show that there was no need to use any additional statistical treatments in our case.

\section{MATERIALS AND METHODS}

Apparatus and conditions: Spectrophotometric measurements were carried out on a computerized Spectronic Gensys 2PC, UV visible $^{-1}$ Spectrophotometer (Milton Roy, USA), using $1.00 \mathrm{~cm}$ quartz cells. The obtained spectral data were saved in PC apparatus program and the subsequent statistical manipulation was performed by transferring the spectral data to Microsoft excel XP program and processing them with the standard curve fit package and matrix calculations. Sonicator (Bransonic 220 BenderHobein $^{-1}$ ), Heater(Salvis) (Heidolph).

Balance (Mettler Toledo). Curve Expert version 1.37 Copyright $^{\circledR} 1995-2001$ by Daniel Hyams. GraphPad Instat version 3.05,32 bit for win $95 \mathrm{NT}^{-1}$ created Sep. 27,2000 Copyright $^{\circledR}$ 1992-2000 by GraphPad software.

Chemicals, reagents and pharmaceutical preparations: All chemicals were of analytical-reagent grade and solvents were of spectrophotometric.

Ibuprofen and paracetamol were kindly provided by the Pharco Co., Egypt. Methanol was purchased 
from Merk (Darmstafd, Germany). Cetafen tablets, Ibuprofen 200, Paracetamo 1325.

Preparation of standard solutions and calibration: Stock standard solutions containing 0.5 and $1.0 \mathrm{mg}$ ibuprofen and paracetamol, respectively, were dissolved in $10 \mathrm{~mL}$ methanol. Standard solutions of both drugs were prepared individually by dilution of the stock solutions with methanol to obtain concentration range of 0.05-5.0 and 0.1-1.0 $\mu \mathrm{g} \mathrm{mL}^{-1}$ for ibuprofen and paracetamol, respectively.

For derivative spectrophotometric method (D1): The values of the D1 amplitudes were measured at $290 \mathrm{~nm}$ (zero-crossing of ibuprofen) and $230 \mathrm{~nm}$ (zero-crossing of paracetamol) for the determination of ibuprofen and paracetamol.

For first derivative of the ratio spectrophotometric method (DD1): According to the theory of the ratiospectra derivative method. The stored UV absorption spectra of standard solutions of ibuprofen were divided wavelength-by-wavelength by a standard spectrum of paracetamol $\left(15 \mu \mathrm{g} \mathrm{mL} \mathrm{mL}^{-1}\right)$. The first derivative was calculated for the obtained spectra with ?? = $3 \mathrm{~nm}$. The amplitudes at $280 \mathrm{~nm}$ was measured and found to be linear to the concentration of ibuprofen. For paracetamol, the stored UV absorption spectra of standard solutions of paracetamol were divided wavelength-by-wavelength by a standard spectrum of ibuprofen $\left(15 \mu \mathrm{g} \mathrm{mL} \mathrm{mL}^{-1}\right)$. The first derivative was calculated for the obtained spectra with $? ?=3 \mathrm{~nm}$. The amplitudes at $270 \mathrm{~nm}$ was measured and found to be linear to the concentration of paracetamol.

For multivariate method: In order to obtain the calibration matrix for applying CLS and PCR analysis, ten solutions of each of the pure components (ibuprofen and paracetamol) were prepared with concentrations in the range 5-60 and $10-100 \mu \mathrm{g} \mathrm{mL}^{-1}$ for ibuprofen and paracetamol, respectively. These ranges were previously verified to obey Beer's law for each of the studied drugs in the selected solvent. The absorption data in the range of 100-40 $\mathrm{nm}$ (digitized every $3.0 \mathrm{~nm}$ ) were subjected to least squares analysis in order to obtain the calibration $\mathrm{K}$ matrix. Laboratory prepared mixtures were prepared by mixing known amounts of ibuprofen and paracetamol in different varied proportions in order to verify the precision of the method for analysis of such mixture and matching the commercial tablets with those having comparable concentrations.
Application to pharmaceutical dosage form: For all methods, 10 tablets labeled to contain * mg of ibuprofen and * mg of paracetamol were powdered. An accurate weight of the powder equivalent to one tablet content was accurately weighed, transferred into a 100 $\mathrm{mL}$ calibrated flask, diluted with methanol, stirred for about $10 \mathrm{~min}$ and then completed to volume with the same solvent. This solution was filtered to remove any insoluble matter. After filtration, working solutions were prepared by transferring suitable aliquots of clear filtrate and diluting with appropriate solvent. The concentrations of the studied drugs were calculated from the corresponding regression equations.

Accuracy study: To study the accuracy of the proposed methods and to check the interference from excipients present in the dosage form, recovery experiments were carried out by the standard addition method.

This study was performed by addition of different amounts of the studied drugs to a known concentration of the pharmaceutical dosage forms.

\section{RESULTS AND DISCUSSION}

D1 method: As shown in Fig. 1 the ultra-violet spectra of standard drugs were found to be overlapped making their simultaneous determination difficult.

In contrast, the D1 spectrum of each pure drug was found to show zero-crossing points (Fig. 2) and assisted in their simultaneous estimation. In practice, the wavelength selected is that which exhibits the best linear response, giving a zero or near zero intercept on the coordinate of the calibration graph and not affected by the presence of any other component. The shape of the first derivative spectra is adequate for determining ibuprofen in the presence of paracetamol and vice versa.

Ibuprofen was determined by measuring of its D1 amplitude at the zero-crossing point of paracetamol (at $230 \mathrm{~nm}$, Fig. 2). While paracetamol was determined by measuring of their D1 at the zero-crossing point of ibuprofen (at $290 \mathrm{~nm}$ for and paracetamol (Fig. 2).

Linear relationships between derivative amplitude and drug concentration were obtained. The linear regression equations together with correlation coefficients slope and intercept, R.S.D. of slope and intercept repeatability (within day) and reproducibility (between-day) obtained for each drug are collected in Table 1 .

DD1 method: The influence of ?? for obtaining the first derivative of the ratio spectra as well as, the effect of divisor concentration on the calibration graphs for 
Am. J. Applied Sci., 5 (8): 1005-1012, 2008

Table 1: Statistical data for the calibration graphs of ibuprofen and paracetamol by 1D, 1DD, CLS and PCR methods

\begin{tabular}{|c|c|c|c|c|c|c|}
\hline & $\begin{array}{l}\text { Linearity range } \\
\left(\mu \mathrm{g} \mathrm{mL}^{-1}\right)\end{array}$ & Slope & Intercept & $\begin{array}{l}\text { Correlation } \\
\text { coefficient }\end{array}$ & R.S.D. & $\mathrm{RSD} \%$ \\
\hline \multicolumn{7}{|l|}{ Ibuprofen } \\
\hline D1 & $5-100$ & 0.057 & -0.006 & 99.70 & 0.60 & 0.85 \\
\hline DD1 & $5-100$ & 0.009 & 0.003 & 100.0 & 0.58 & 0.87 \\
\hline CLS & $1-10$ & 0.857 & 0.030 & 99.94 & 0.68 & 0.49 \\
\hline PCR & $1-6$ & 0.895 & 0.102 & 99.53 & 0.90 & 0.73 \\
\hline \multicolumn{7}{|l|}{ Paracetamol } \\
\hline D1 & $10-100$ & 0.054 & 0.003 & 100.1 & 0.87 & 0.48 \\
\hline DD1 & $10-100$ & 0.005 & 0.003 & 100.2 & 0.81 & 0.54 \\
\hline CLS & $2-10$ & 0.594 & 0.093 & 100.2 & 0.59 & 0.46 \\
\hline PCR & $2-6$ & 0.709 & 0.083 & 100.1 & 0.60 & 0.54 \\
\hline
\end{tabular}

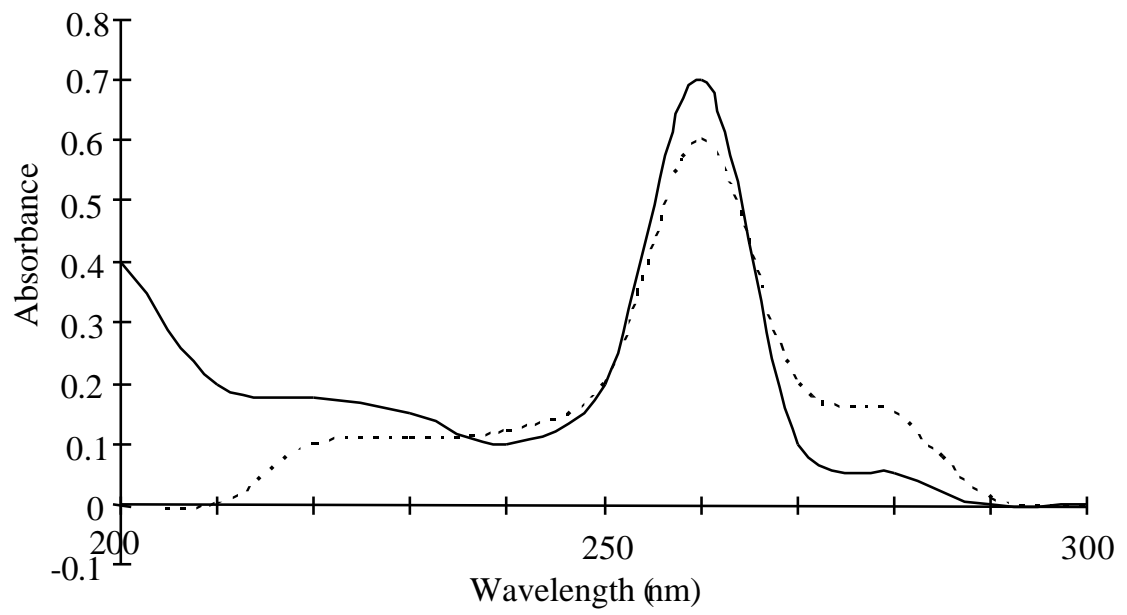

Figure 1: Absorption spectra of Ibuprofen $(-, 5 \mathrm{ug})$ and paracetamol $(\cdots \cdots \cdots, 5 \mathrm{ug})$

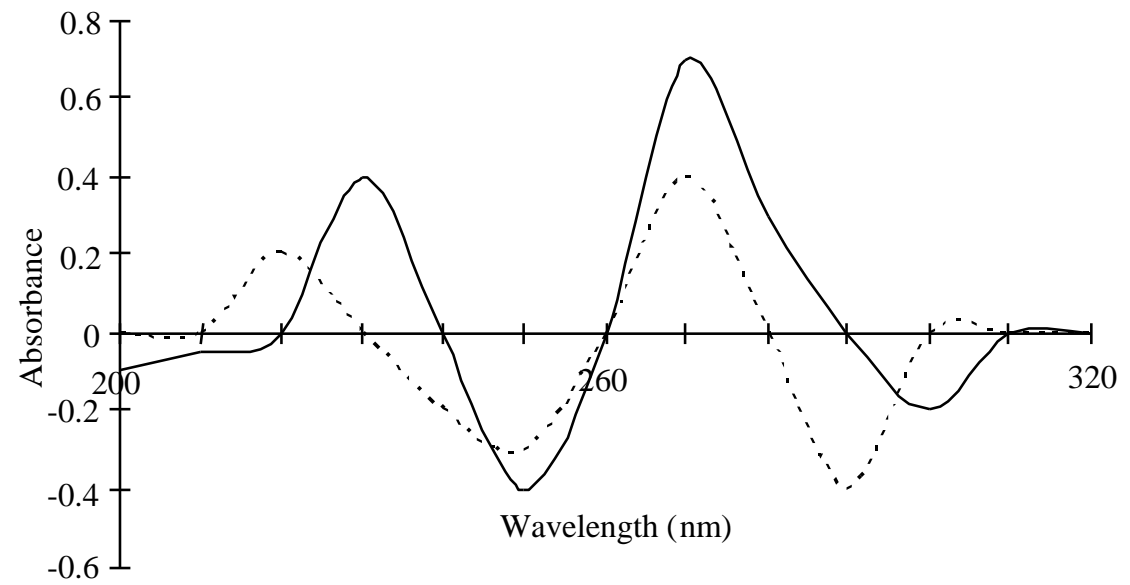

Fig. 2: First order bsorption spectra of Ibuprofen $(-, 5 \mathrm{ug})$ and paracetamol $(\cdots \cdots \cdots, 5 \mathrm{ug})$ 


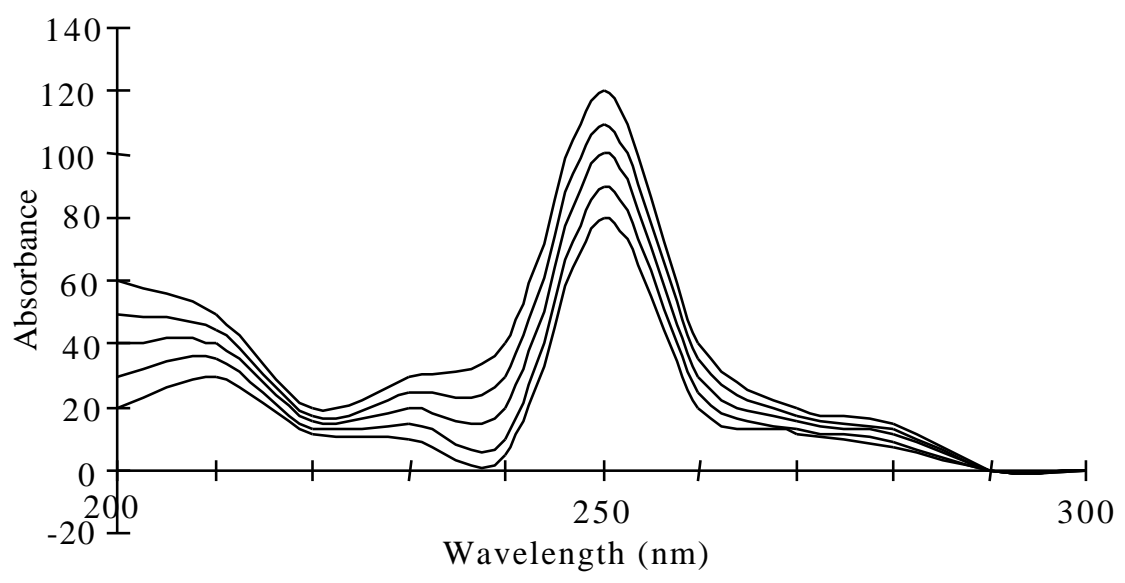

Fig. 3: Absorption spectra of ibuprofen and paracetamol as divisor

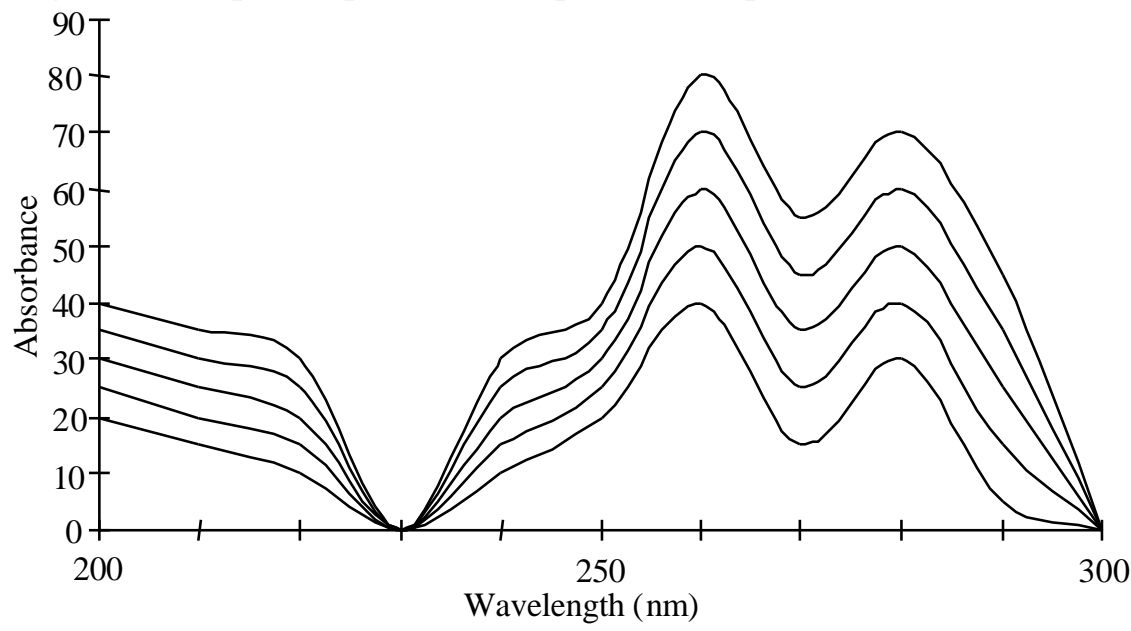

Fig. 4: Absorption spectra of paracetamol and ibuprofen as divisor

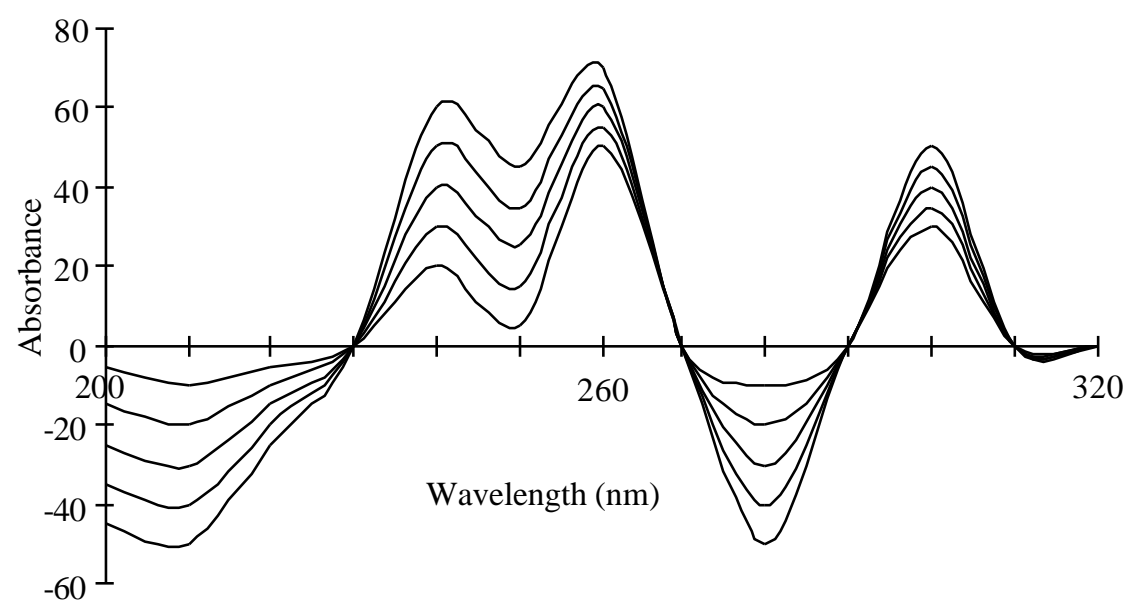

Fig. 5: First order of absorption spectra of ibuprofen and paracetamol as divisor 1009 


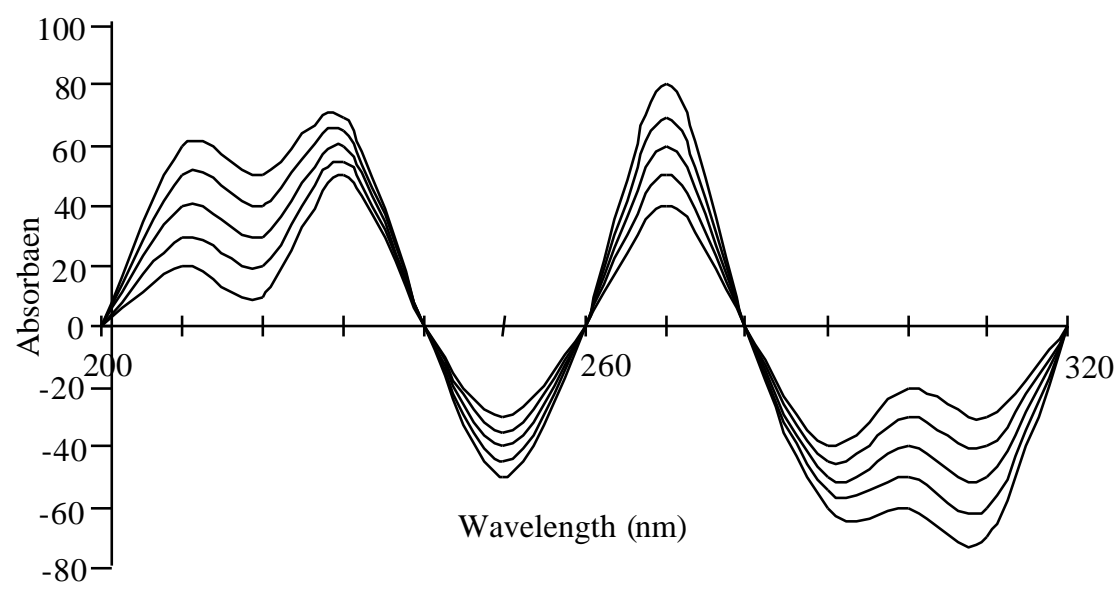

Fig. 6: First order of absorption spectra of paracetamol and ibuprofen as divisor

the proposed mixture was studied in order to select the best factors affecting the determination. Results indicated that ? $?=3 \mathrm{~nm}$ was most suitable one, while the divisor concentration has no significant effect on the assay results for the studied mixtures.

For determination of ibuprofen, the absorption spectra of ibuprofen were divided by that of standard solution of either paracetamol $\left(15 \mu \mathrm{g} \mathrm{mL}^{-1}\right)$ (Fig. 3) and the absorption spectra of paracetamol were divided by that of standard solution of ibuprofen $\left(6 \mu \mathrm{g} \mathrm{mL}{ }^{-1}\right)$, (Fig. 4).

The first derivative of the developed ratio spectra were calculated with ? $=3 \mathrm{~nm}$. Figure 5 shows that, ibuprofen can be determined by measuring the amplitude at many wavelengths where paracetamol has no contribution, but it was found that the amplitude at $280 \mathrm{~nm}$ give the most accurate and sensitive results for ibuprofen in presence of paracetamol (Fig. 5). Figure 6, shows that paracetamol can be determined by measuring the amplitude at many wavelengths where ibuprofen have no contribution.

The proposed methods are applicable over the ranges $* *_{-} * * *$ to $* *_{-} * * * \mu \mathrm{g} \mathrm{mL} \mathrm{m}^{-1}$ for ibuprofen and paracetamol. The characteristic parameters and necessary statistical data of the regression equations and reproducibility data are collected in Table 1.

Repeatability and reproducibility variables were characterized by R.S.D. (\%) and by the difference between theoretical and measured concentrations. There was no significant difference for the assay, which was tested within-day (repeatability) and between-days (reproducibility).

In order to demonstrate the validity and applicability of the proposed DD1 method, recovery studies were performed by analyzing laboratory prepared mixtures with different composition ratio (Table 2).

Multivariate method: The absorption spectra of the studied drugs are shown in Fig. 1. As could be seen, a considerable degree of spectral overlapping occurs in the region from 200 to $300 \mathrm{~nm}$ for the studied drugs. The degree of spectral overlapping was given by $\left(D_{i}\right)^{0.5}$. In case of the presently studied compounds, the spectra lead to $\mathrm{D}=0.50$ implying $* * * * \%$ for the studied mixture.

Several laboratory prepared mixtures were subjected to the CLS and PCR analysis in order to confirm the suitability of the calibration model for determination of the studied drugs in the pharmaceutical sample solutions.

The results of commercial dosage form and laboratory prepared mixtures analysis with comparable concentrations were found closely matched. This indicated that, excipients and additives did not interfere with the determinations. Moreover, the results of dosage form analysis were compared with those obtained by applying reported method. As shown in Table 2, the results are in good agreement with those of the reported procedure as indicated by the calculated $t$ and $F$ values.

Application to pharmaceutical dosage form: The three proposed methods are successfully applied for the simultaneous determination of Cortefen tablets without interference of the excipients present and without prior separation. The utility of the three proposed methods were verified by replicate estimations of the pharmaceutical preparations and results obtained were evaluated statistically (Table 2). 
Am. J. Applied Sci., 5 (8): 1005-1012, 2008

Table 2: Determination of ibuprofen and paracetamol in authentic, laboratory prepared mixtures and pharmaceutical dosage form using D1, DD1, CLS, PCR and reported methods

\begin{tabular}{|c|c|c|c|c|c|}
\hline Standard solution & Reported & D1 & DD1 & CLS & PCR \\
\hline Authentic ibuprofen & $99.7 \pm 0.37$ & $\begin{array}{l}99.8 \pm 0.40 \\
t=0.88 \\
F=1.14\end{array}$ & $\begin{array}{l}100.1 \pm 0.40 \\
t=1.78 \\
F=1.14\end{array}$ & $\begin{array}{l}99.9 \pm 0.39 \\
t=0.88 \\
F=1.10\end{array}$ & $\begin{array}{l}100.0 \pm 0.41 \\
t=1.33 \\
F=1.20\end{array}$ \\
\hline Authentic paracetamol & $99.9 \pm 0.72$ & $\begin{array}{l}100.0 \pm 0.70 \\
t=0.21 \\
F=1.03\end{array}$ & $\begin{array}{l}101.1 \pm 0.71 \\
t=0.42 \\
F=1.01\end{array}$ & $\begin{array}{l}99.8 \pm 0.7 \\
t=0.21 \\
F=1.01\end{array}$ & $\begin{array}{l}100.1 \pm 0.71 \\
\mathrm{t}=0.42 \\
\mathrm{~F}=1.01\end{array}$ \\
\hline $\begin{array}{l}\text { Laboratory prepaed mix. } \\
\text { for ibuprofen }\end{array}$ & $100.0 \pm 0.65$ & $\begin{array}{l}99.9 \pm 0.66 \\
t=0.53 \\
F=1.05\end{array}$ & $\begin{array}{l}99.8 \pm 0.65 \\
t=0.47 \\
F=1.35\end{array}$ & $\begin{array}{l}99.9 \pm 0.50 \\
t=0.55 \\
F=1.68\end{array}$ & $\begin{array}{l}100.0 \pm 0.73 \\
\mathrm{t}=0.49 \\
\mathrm{~F}=1.26\end{array}$ \\
\hline $\begin{array}{l}\text { Laboratory prepared mix. } \\
\text { for paracetamol }\end{array}$ & $100.1 \pm 0.39$ & $\begin{array}{l}99.8 \pm 0.40 \\
t=0.83 \\
F=1.03\end{array}$ & $\begin{array}{l}100.0 \pm 0.38 \\
t=0.28 \\
F=1.03\end{array}$ & $\begin{array}{l}100.2 \pm 0.39 \\
t=0.62 \\
F=1.00\end{array}$ & $\begin{array}{l}99.90 .41 \\
t=0.55 \\
F=1.00\end{array}$ \\
\hline $\begin{array}{l}\text { Cetafen tablets. } \\
\text { for ibuprofen }\end{array}$ & $99.6 \pm 0.53$ & $\begin{array}{l}99.8 \pm 0.50 \\
t=0.47 \\
F=1.06\end{array}$ & $\begin{array}{l}99.7 \pm 0.56 \\
t=0.24 \\
F=1.06\end{array}$ & $\begin{array}{l}99.9 \pm 0.55 \\
t=0.71 \\
F=1.04\end{array}$ & $\begin{array}{l}99.8 \pm 0.54 \\
t=0.47 \\
F=1.02\end{array}$ \\
\hline $\begin{array}{l}\text { Cetafen tablets } \\
\text { for paracetamol }\end{array}$ & $100.2 \pm 0.75$ & $\begin{array}{l}100.1 \pm 0.69 \\
t=0.24 \\
F=1.17\end{array}$ & $\begin{array}{l}100.0 \pm 0.75 \\
t=0.47 \\
F=1.00\end{array}$ & $\begin{array}{l}100.0 \pm 0.77 \\
t=0.45 \\
F=1.05\end{array}$ & $\begin{array}{l}99.9 \pm 0.80 \\
t=0.68 \\
F=1.14\end{array}$ \\
\hline
\end{tabular}

Theoretical values at $95 \%$ confidence limit are $\mathrm{t}=3.58$ and $\mathrm{F}=4.28$

Validity of the proposed methods: Statistical analysis of the results obtained for the proposed methods (Table 2), shows that all the suggested measurements are equally precise and accurate to the reported methods.

\section{CONCLUSION}

The D1and DD1 and multivariate (CLS and PCR) methods enable the quantitation of ibuprofen and paracetamol binary mixture with good accuracy and precision, either in laboratory prepared samples or in pharmaceutical dosage forms. By comparing the results of the three proposed methods, no significant difference is obtained. All of the proposed procedures have the advantage of being rapid, precise and direct. DD1 method has two advantages over the D1 method, the first is the high sensitivity and accuracy, the second is the easy measurements at any peak signal giving higher values. Classical least squares and PCR multivariate calibration analysis were developed for the analysis of the laboratory prepared mixtures and pharmaceutical dosage forms. The good recoveries obtained in all cases as well as the reliable agreement with the reported procedures proved that, the proposed methods could be applied efficiently for determination of ibuprofen and paracetamol binary mixture with quite satisfactory precision and could be easily used in a quality control laboratory for their analysis.

\section{REFERENCES}

1. Martindale,(2002) The Complete Drug Reference; $33^{\text {rd }}$ Ed., pharmaceutical press, London.

2. Haikala, V. E. , Heimonen, I.K., Vuorela, H.J., 1991.Determination of ibuprofen in ointments by reversed-phase liquid chromatography. J. of pharmaceutical sciences, 80(5), 456-458.
3. Sodhi, R.A., Chawla, J.L., San, R.T., 1996. Simultaneous determination of paracetamol, ibuprofen and chorzoxazone by HPLC, HPTLC and GC methods. Indian Drugs, 33(6), 280-285.

4. Zarapkar, S.S., Halkar, U.P., Bhandari, N.P., 1999. Reverse phase high performance liquid chromatographic determination of Ibuprofen, Paracetamol and Methocatbamol in tablets. Indian drugs, 36(11) 710-713.

5. Li, J. Gao, Y.-H., Gao, Y. S., Li, X.G., 2000. Simultaneous determination of ibuprofen and pseudoephedrine in ibuprofen and pseudoephedrine hydrochloride granules by HPLC assay. Chinese pharmaceutical J. 35(9), 623-624.

6. Joanna Lenik, Barbara Marczewska, Cecylia Wardak, 2004. Properties of ion-selective electrodes with polymer membranes for ibuprofen determination. Desalination, 163, 77-83.

7. Santini, A.O., de Oliveira, J. E., Pezza, H.R., pezza, L., 2006. A new potentiometric ibuprofen ion sensor immobilized in a graphite matrix for determination of ibuprofen in tablets. Microchemical J., 84(1-2), 44-49.

8. Singhvi, I., Chaturvedi, S.C. 1998. Spectrophotometric methods for simultaneous estimation of ibuprofen and pseudoephedrine hydrochloride from tablets. Indian Drugs 35(4), 234-238.

9. Sachan, A., Trivedi, P., 1998, First derivative spectrophotometric estimation of ibuprofen and dextropropoxyphene hydrochloride in solid dosage formulations. Indian Drugs 35 (12) 762-765. 
10. Ivanovic, D., Medenica, M., Markovic, S., Mandic, G., 2000. Second-derivative spectrophotometric assay of pseudoephedrine, ibuprofen and loratadine in pharmaceuticals . Arzneimittel-Forschung/ Drug Research. 50 (11), 1004-1008.

11. Damiani, P. C., Bearzotti, M., Cabezon, M. A.,2001. Spectrofluorometric determination of ibuprofen in pharmaceutical formulations. J. of pharmaceutical and Biomedical analysis 25 (3-4), 679-683.

12. Teslyuk, O.I., Bel'tyukova, S.V., Yegorova A.V. Yagodkin, B.N., 2007. Complex compounds of terbium(III) with some nonsteroidal antiinflammatory drugs and their analytical applications. J. of Analytical Chemistry 62 (4) 330-335.

13. Sena, M.M., Freitas, C.B. Silva, L.C, Perez, C.N., De paula, Y.O., 2007. Simultaneous spectrophotometric determination of paracetamol and ibuprofen in pharmaceutical formulation by multivariate calibration. Quimica Nova 30 (1), 7579.

14. Emre, D., Ozaltin, N., 2007. ibuprofen determination of paracetamol, caffeine and propyphenazone in ternary mixtures by micellar electrokinetic capillary chromatography. J. Of chromatography B: Analytical Technologies in the Biomedical and Life Sciences 847 (2), 126-132.

15. Gopinath, R. Rajan, S., Meyyanathan, S.N., Krishnaveni, N., Suresh, B., 2007. A RP-HPLC method for simultaneous estimation of paracetamol and aceclofenac in tablets. Indian $\mathrm{J}$. of pharmaceutical sciences 69 (1), 137-140.

16. Senthamil Selvan, P., Gopinath, R., Saravanan, V.S., Gopal, N., Sarvana Kumar, A., periyasamy, K., 2007. Simultaneous estimation of paracetamol and aceclofenac in combined dosage forms by RPHPLC method. Asian J. of chemistry. 19 (2), 10041010 .

17. Suntornsuk, L., Pipitharome, O., and Wilairat, P., 2003, J. pharm. Biomed. Anal, 33(3), 441.

18. Ni, Y., Wang, Y., Kokot, S., 2004. Differential pulse stripping voltammetric determination of paracetamol and Phenobarbital in pharmaceuticals assisted by chemometrices. Analytical Letters 37, 3219-3235.

19. Azhagvuel, S., Sekar, R. 2007, Method development and validation for the simultaneous determination of cetrizine dihydrochloride, paracetamol, and phenylpropanolamine hydrochloride in tablets by capillary zone electrophoresis. J. of Pharmaceutical and Biomedical Analysis 43 (3), 873-878.

20. Burakham, R., Duangthong, S., Patimapornlert, L. Lenghor, N., Kasiwad, S., Srivichai, L., Lapanantnoppakhun, S., Jakmunee, J., Grudpan, K., 2004. Flow-injection and sequential-injection determinations of paracetamol in pharmaceutical preparations using nitrosation reaction. Analytical Sciences. 20 (5), 837-840.
21. De Los A. Oliva, M., Olsina, R.A., Masi, A.N., 2005. Selective spectrofluorimetric method for paracetamol determination through coumarinic compound formation. Talanta 66 (1) 229-235.

22. Lavorante, A.F., Pires, C.K, Reis, B.F., 2006. Multicommuted flow system employing pinch solenoid valves and micro-pumps. Spectrophotometric determination of paracetamol in pharmaceutical formulations. J. of Pharmaceutical and Biomedical analysis 42 (4), 423-429.

23. Berzas Nevado, J.J.; Rodriguez Flores, J.; Villasenor Llerena, M.J.1993, Simultaneous determination of tartazine and sunset yellow by derivative spectrophotometry and ratio spectra derivative. Talanta 40(9), 1391-1396.

24. Bonazzi, D., Gotti, R., Andrisano, V., and Cavrini, V., 1996. Farmaco, 51(11), 733.

25. Wehner, W., 2000 pharmazie 55(0), 543.

26. Ferraro, M.C.F., Castellano, P.M., and Kaufman, T.S., 2003. Anal. Bioanl. Chem. 377 (7-8), 1159. Through analytical abstract 66(4); G68.

27. Blanco M., Gene J., Iturriaga H., Maspoch S. and Riba J., 1987.. Talanta, 34, 987.

28. Mohamed Abdel-Kawy, Sawsan M. Amer, Hayam M. Lotfy and Hala E. Zaazaa., 2005, Determination of atenolol, chlorothalidone and amiloeide in combinations Bull. Fac. Pharm. Cairo Univ., 43(2), 51-63.

29. Hayam Mohmoud Lotfy., 2006 Simultaneous determination of omeprazole, tinidazole and clarithromycin in combination Bull. Fac. Pharm. Cairo Univ., 44(2), 27-39.

30. Adam, M.J., 2004 Chemometrics in analytical chemistry". $2^{\text {nd }}$ Edition, Alden Book set, Northampton, U.K, Copyright ${ }^{\circledR}$ 177-181.

31. Wetzel D.L, 1993, Anal. Chem., 55, 1165 A.

32. Beebe, K.R. and Kowalski B.R., 1987, An introduction to multivariate calibration and analysis. Anal. Chem., 59(17), 1007.

33. Haaland, D.M., and Thomas, E.V., 1988, Partial least-squares methods for spectral analyses. 1.Relation to other quantitative calibration methods and the extraction of qualitative information. Anal. Chem., 60(11), 1193.

34. Thomas, E.V. and Haaland, D.M., 1990 Anal. Chem. 62, 1090.

35. Martens, H. and Naes, T., 1991 " Multivariate Calibration" ,John Willy and Sons, New York. Bent Jorgensen, 2003. Multivariate data analysis and chemometrices throug 